\title{
Results from the ARGO-YBJ experiment
}

\author{
Roberto luppa for the ARGO-YBJ collaboration*广 \\ University of Rome Tor Vergata \\ INFN Sez.ne di Roma Tor Vergata \\ E-mail: roberto.iuppaeroma2.infn.it
}

The ARGO-YBJ experiment consists of a $5000 \mathrm{~m}^{2}$ single layer of Resistive Plate Chambers situated at Yangbajing, Tibet (P.R. of China), 4300 meters a.s.l. (atmospheric depth $600 \mathrm{~g} / \mathrm{cm}^{2}$ ). The percentage of active area all over the carpet is $92 \%$ and a partially instrumented guard ring $\left(1700 \mathrm{~m}^{2}\right)$ around the central zone extends the instrumented area up to $11000 \mathrm{~m}^{2}$.

The experiment is operated in scaler mode, shower mode and analog mode and is sensitive to cosmic rays in the $10^{9}-10^{15} \mathrm{eV}$ energy range.

From few hundreds $\mathrm{GeV}$ on, the arrival direction of cosmic rays is inferred by the study of the induced extensive air showers. A review of the most important results of the ARGO-YBJ experiment in gamma-ray astronomy and cosmic-ray physics will be given, focusing on the potential of RPCs in ground-based astroparticle physics.

XI workshop on Resistive Plate Chambers and Related Detectors - RCP2012,

February 5-10, 2012

INFN Laboratori Nazionali di Frascati Italy

\footnotetext{
* Speaker.

${ }^{\dagger}$ The author is indebted with Prof. R. Santonico for his contribution to the idea of this document.
} 


\section{Introduction}

In the last few years, astrophysics enjoyed the observative contribution of more and more refined telescopes, sensitive from the radio to the gamma region of the electromagnetic spectrum. On the other side, cosmic-ray ( $\mathrm{CR}$ ) physics benefited from the unexpected findings in the wide energy range spanning $10^{6}$ to $10^{21} \mathrm{eV}$.

The energy region $10^{9}-10^{15} \mathrm{eV}$ is important for the study of the origin and the propagation of CRs, due to the direct relation that there exists between charged nuclei of such energy and the emission spectra observed from many celestial objects, either galactic and extra-galactic. At this energy, fluxes are so low that large collecting areas are needed and ground-based detectors are to be used. Extensive Air Shower (EAS) arrays detect secondary particles of the shower front and use the time-space information to inspect the primary CR that produced the shower. For gamma-ray astrophysics, an accurate reconstruction of the shower parameters is needed, i.e. nanosecond accuracy in sampling the arrival times of the shower front particles. Moreover, to efficiently detect showers induced by primary CRs of energy as low as $\sim 10^{11} \mathrm{eV}$, the absorption of the atmosphere must be as low as possible and as much secondary particles as possible are to be detected. The first condition is satisfied by choosing high-altitude sites for the experiments, whereas the second one naturally implies the full-coverage approach, i.e. to build detectors whose active area is few less than $100 \%$. Such a concept demands robust, reliable and cost-effective detection technologies. In the late 1990s, the use of Resistive Plate Chambers (RPCs) was envisaged to meet these requirements by the ARGO-YBJ collaboration, due to RPC low-cost, large active area and excellent time resolution [1]. The ARGO-YBJ experiment was mainly designed for gamma-ray astro-physics in the energy range $\sim 10^{9}-10^{13} \mathrm{eV}$ and $\mathrm{CR}$ physics in the rigidity range $10^{12}-10^{16} \mathrm{~V}$.

This paper is aimed at giving a summary of the main achievements of the ARGO-YBJ eXperiment, mainly focusing on those results which follow from the particular features of the RPC detector.

\section{The ARGO-YBJ detector}

The ARGO-YBJ experiment is a continuous carpet of RPC deployed at the YangBaJing Cosmic Ray Laboratory, Tibet, P.R. of China, $4300 \mathrm{~m}$ a.s.l. $\quad(\sim$ $\left.606 \mathrm{~g} / \mathrm{cm}^{2}\right)$. The detector is constituted by a central carpet $\sim 74 \times$ $78 \mathrm{~m}^{2}$, made of a single layer of RPCs with $\sim 93 \%$ of active area, enclosed by a guard ring partially instrumented $(\sim 20 \%)$ up to $\sim 100 \times 110 \mathrm{~m}^{2}$.

The apparatus has a modular structure (see the figure 1), the ba-

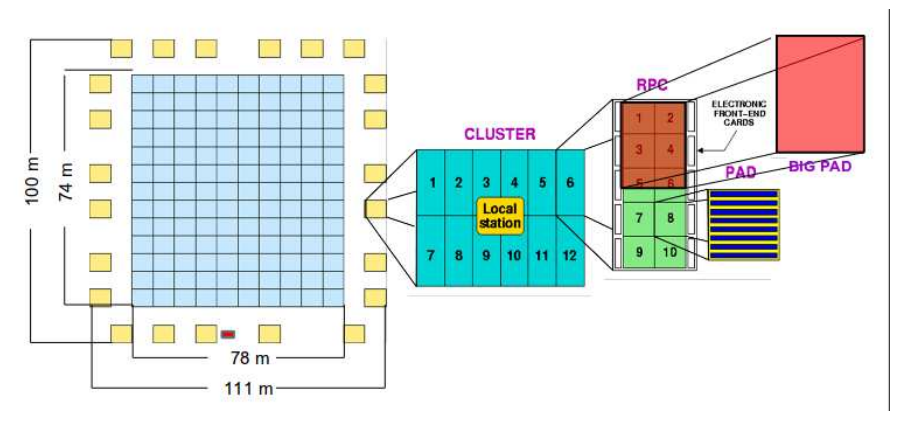

Figure 1: Layout of the ARGO-YBJ experiment. On the left side the central carpet (cyan) and the guard ring (yellow) are represented. The small red rectangle indicates the position of the testtelescope used to monitor the RPC performance. The modular structure of the detector is showed in the right side of the figure. The basic DAQ unit is the cluster, made of 10 RPCs. Time and space pixels (pad and strip, respectively) are also visible, as well as the big-pad geometry for the analog read-out. 
sic data acquisition element being

a cluster $\left(5.7 \times 7.6 \mathrm{~m}^{2}\right)$, made of $12 \mathrm{RPCs}\left(2.85 \times 1.23 \mathrm{~m}^{2}\right.$ each). Each chamber is read by $80 \mathrm{ex}$ ternal strips of $6.75 \times 61.8 \mathrm{~cm}^{2}$ (the spatial pixel), logically organized in 10 independent pads of $55.6 \times 61.8 \mathrm{~cm}^{2}$ which represent the time pixel of the detector [2]. The read-out of 18360 pads and 146880 strips are the experimental output of the detector.

The RPCs are operated in streamer mode by using a gas mixture (Ar 15\%, Isobutane $10 \%$, TetraFluoroEthane 75\%) for high altitude operation [1]. The high voltage settled at $7.2 \mathrm{kV}$ ensures an overall efficiency of about $96 \%$. The intense field of $3.6 \mathrm{kV} / \mathrm{mm}$ at $0.6 \mathrm{~atm}$ pressure provides very good time resolution $\left(1.8 \mathrm{~ns}\right.$ ) and the high electrode resistivity (plastic laminate, $10^{11} \Omega \mathrm{cm}$ ) limits the area interested by the electrical discharge to few $\mathrm{mm}^{2}$.

A simple, yet powerful, electronic logic has been implemented to build an inclusive trigger. This logic is based on a time correlation between the pad signals depending on their relative distance. In this way, all the shower events giving a number of fired pads $\mathrm{N}_{\text {pad }} \geq \mathrm{N}_{\text {trig }}=20$ in the central carpet in a time window of 420 ns generate the trigger.

The whole system, in smooth data taking since July 2006 with ARGO-130, is in stable data taking with the full apparatus of 153 clusters since November 2007 with the trigger condition $\mathrm{N}_{\text {trig }}$ $=20$ and a duty cycle $\geq 85 \%$. The trigger rate is $\sim 3.5 \mathrm{kHz}$ with a dead time of $4 \%$.

Once the coincidence of the secondary particles has been recorded, the main parameters of the detected shower are reconstructed from the digital information, by following the procedure described in [3]: among them, the shower size (proportional to the primary CR energy) and the arrival direction. Since December 2009, the analog information about the event is recorded too.

\section{Performance}

The operational performance of the apparatus is continuously monitored by a test-telescope (see the figure 1) operated with the whole experiment. The efficiency and the time resolution are measured for three distinct RPCs and are interpreted as average values for the whole system.

In figure 2 the time resolution and the efficiency from the test telescope are reported for the period 2008-2011. 1 year-periodic variations
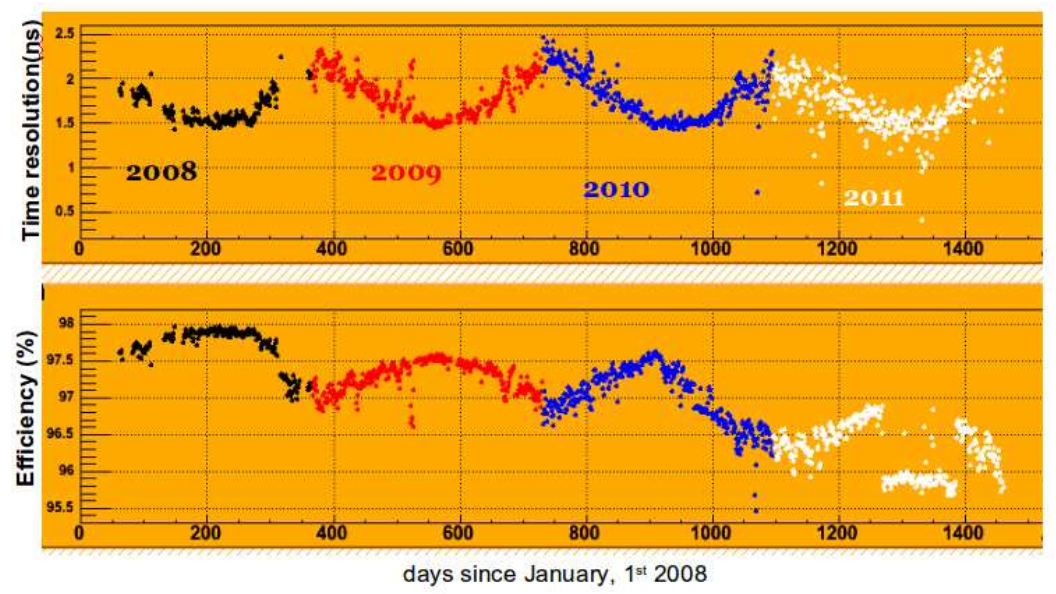

Figure 2: Time resolution (upper plot) and efficiency (lower plot) of the ARGO-YBJ RPCs obtained from the test-telescope. Seasonal variations induced by atmospheric effects are well visible. The reported efficiency does not account for the blind area of the spacers inside the gas gap. The efficiency drop in the mid part of 2011 is due to High Voltage tests. 

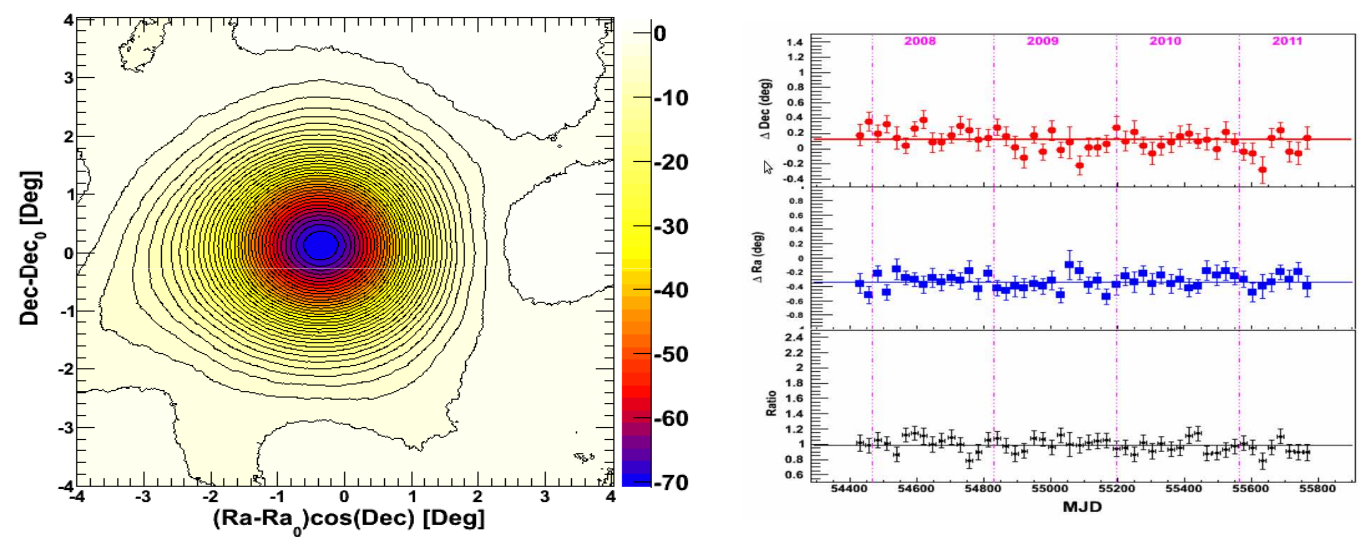

Figure 3: Moon shadow as observed by the ARGO-YBJ experiment (left plot). The color scale indicates the significance of the detection in standard deviations. The right plot reports some results about the operational stability of ARGO-YBJ, carried out on a monthly basis. The deviation of the Moon signal from the expected position in declination (red) and right ascension (blue points) is reported. The black points indicate the ration of the measured signal intensity to the expected one.

the time resolution (upper plot) and for the efficiency (lower plot). They are induced by atmospheric effects, as no correction of the applied electric field is made for temperature and pressure changes. After these effects, the intrinsic operating stability was measured to be at the level of $0.5 \%$. Besides such a seasonal effect, the efficiency plot shows a small decrease with the time, as large as $3 \times 10^{-3}$. It is consistent with the expected aging effects for RPCs operated in streamer regime.

The Moon shadow. The performance of the detector can be also measured with the Moon shadow, i.e. by observing the $\mathrm{CR}$ deficit towards the Moon (figure 3, left). It allows to evaluate the angular resolution, the pointing accuracy and to energy-calibrate the detector, as well as to check its operation stability [3].

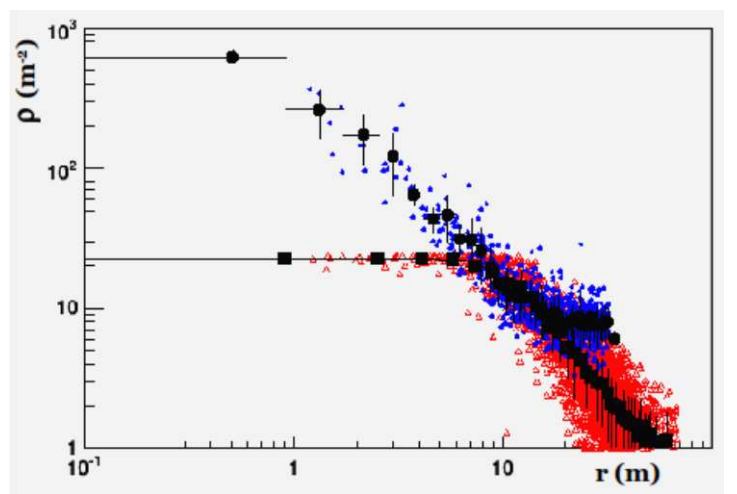

Figure 4: Example of inter-calibration of the digital and the analog information within the same event.
Analog read-out. Since December 2009 the ARGO-YBJ experiment started taking data also in analog mode, i.e. by reading the charge deposit on each big-pad. The ana$\log$ information allows to ARGO-YBJ to overtake the limit of the digital saturation, extending the energy range of operation up to $\sim 10 \mathrm{PeV}$. More details can be found in [4]. In the figure 4 an instance of calibration of the analog readout on an event-by-event basis is given. The vertical axis reports the particle density, whereas the horizontal one indicates the distance from the reconstructed position of the shower core. Red points represent the digital information, saturating at $\sim 22 \mathrm{part} / \mathrm{m}^{2}$. The analog signal, represented by the blue points, 


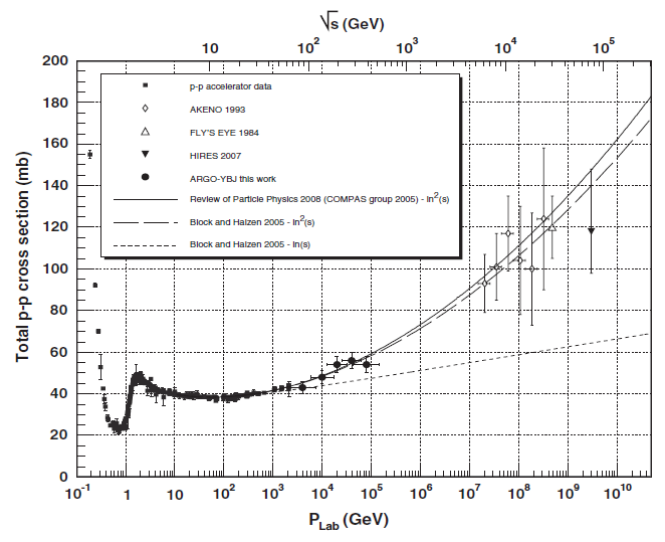

(a)

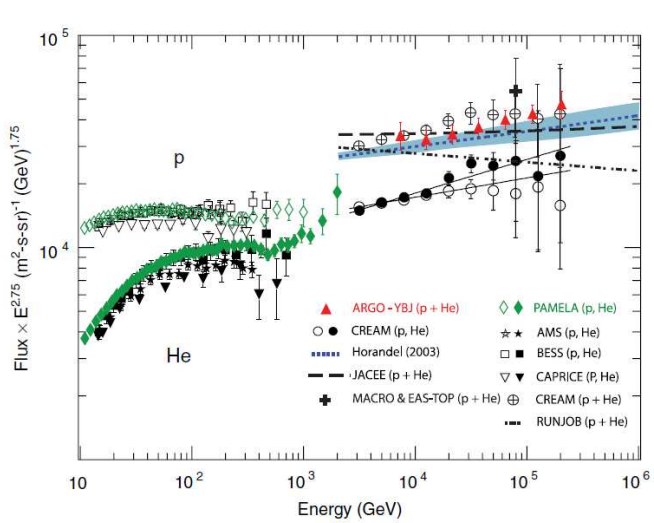

(b)

Figure 5: (a): p-ptotal cross-section measured by the ARGO-YBJ experiment. LHC experiments confirmed in the last year. (b): light component ( $\mathrm{p}+\mathrm{He}$ ) energy-spectrum as measured by ARGO-YBJ. Heavier elements amount to a few percent of the data-set and can be reliably excluded from the analysis by suitable selection cuts.

gives significant information up to $\sim 10^{3} \mathrm{part} / \mathrm{m}^{2}$. In the region between 5 and $20 \mathrm{~m}$ from the shower core, digital and analog distribution perfectly overlap with each other. The use of RPCs in analog mode on large-scale experiments has no precedent and currently gives promising results.

\section{Physics results}

This section reports a selection of those results from ARGO-YBJ which better highlight how and where RPCs are most effective for astroparticle physics.

p-p total cross-section. The figure 5(a) reports the measurement of the total p-p cross section as measured by ARGO-YBJ [5]. The result dates back to 2009 and is obtained by exploiting the cosmic ray intensity attenuation observed with increasing angle between the arrival direction of primary cosmic rays and the zenith. Such a measurement is traditionally carried out by combining the information on the muonic and the electromagnetic content of the shower. For ARGO-YBJ the muon information, not available, was replaced by the information on the local particle density, which can be made use of only if most of the shower front is sampled.

Light-component energy spectrum. In the figure 5(b) the energy spectrum of the light component of the cosmic radiation is reported. It is the final outcome of the analysis described in [6]. It is worth noting that all the other points in the plot are from satellite- or balloon-borne experiments. The full coverage approach and the high efficiency of RPCs for low-energy particles allowed ARGO-YBJ to be so sensitive in the TeV energy region.

Mrk421 long-term monitoring. The figure 6(a) reports the cumulative number of photons coming from the Active Galactic Nucleus Mrk421 as a function of time. Photons are emitted with high variability,as it is well evident from the variation of the slope of the red band. For these objects long-term monitoring activities have fundamental importance, mostly for what concerns multiwavelength observation campaigns. ARGO-YBJ is currently the only experiment with duty-cycle sufficient $(>85 \%)$ to guarantee a continuous survey from $\sim 300 \mathrm{GeV}$ to $\sim 20 \mathrm{TeV}$ on a large field of view ( $\sim 2 \mathrm{sr}$ in the local frame). More details can be found in [7]. 


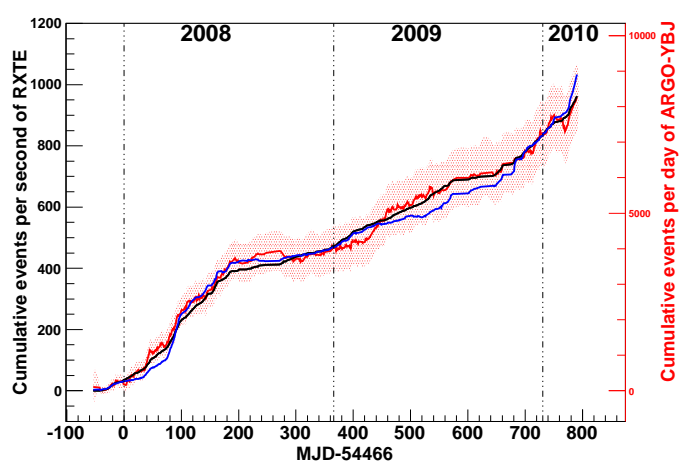

(a)

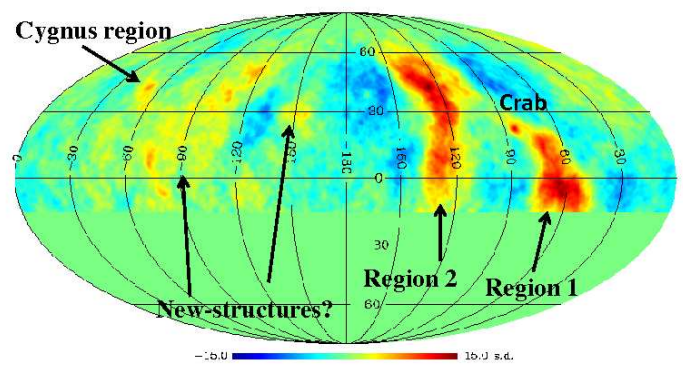

(b)

Figure 6: (a): Mrk421 long-term monitoring by the ARGO-YBJ experiment. The vertical axis reports the cumulative number of ARGO-YBJ events (red band, statistical error only), together with two correlated cumulative distributions of X-ray emission. (b): Medium scale anisotropy observed by the ARGO-YBJ experiment. An equatorial representation of data is provided. The color scale indicates the statistical significance of the observation.

Medium scale anisotropy. The Medium Scale Anisotropy of cosmic rays was observed by ARGO-YBJ in the energy range $600 \mathrm{GeV}-20 \mathrm{TeV}$ (figure 6(b)). Cosmic ray excesses narrow down to $10^{\circ}$ are a recent discovery. Even if the intensity of the effect reported here is of the order of $10^{-4}-10^{3}$, the response uniformity of RPCs after temperature and pressure corrections allows the ARGO-YBJ collaboration to reduce systematics down to $20 \%$. More details can be found in [8].

\section{Conclusions}

The ARGO-YBJ experiment is operated since November 2007 with duty cycle $>85 \%$ and trigger rate $\sim 3.5 \mathrm{kHz}$. Since December 2009 the analog readout extends the energy range of the experiment up to $\sim 10 \mathrm{PeV}$. The RPC technology demonstrated to be effective with respect to the main experimental challenges of ground-based $\mathrm{TeV}$ astroparticle physics.

\section{References}

[1] Bacci et al., High Altitude test of RPCs for the ARGO-YBJ experiment, NIM A 443 (2000) 342-350

[2] Aielli et al., Layout and performance of RPCs used in the Argo-YBJ experiment, NIM A 562 (2006) 92-96

[3] Bartoli et al.,Observation of the cosmic ray moon shadowing effect with the ARGO-YBJ experiment., PRD 84 (2011) 022003

[4] Aielli et al., Calibration of the RPC charge readout in the ARGO-YBJ experiment., NIM A 661 (2012) S56

[5] Aielli et al., Proton-air cross section measurement with the ARGO-YBJ cosmic ray experiment., PRD 80 (2009) 092004

[6] Bartoli et al., Light-component spectrum of the primary cosmic rays in the multi-TeV region measured by the ARGO-YBJ experiment., PRD 85 (2012) 092005

[7] Bartoli et al., Long-term monitor of Mrk 421 TeV emission using ARGO-YBJ experiment., ApJ 734 (2011) 110

[8] R. Iuppa for the ARGO-YBJ collaboration, Anisotropies in the cosmic radiation observed with $A R G O-Y B J$, in 2011 Fermi symposium proceedings - eConf C110509 (2011), arXiv:1112.2375 